\title{
VALORES INTERPERSONALES EN ESTUDIANTES DEL I Y VIII CICLO ACADÉMICO DE UNA UNIVERSIDAD PRIVADA
}

\author{
Teresa Del Pilar García García*
}

\begin{abstract}
(19)
Resumen

La presente investigación permite conocer el impacto del Eje Transversal de Ética y Valores, en el desarrollo de los valores interpersonales en los estudiantes del primero y octavo ciclo de una universidad privada. El citado eje es complementario a la formación académica en aspectos relacionados a los valores como solidaridad, liderazgo, soporte, entre otros; y tiene como objetivo contribuir a la formación humana. Para fines de la investigación se utilizó el Cuestionario de Valores Interpersonales de Leonard Gordon (Survey of Interpersonal Values SIV), siendo aplicado a una muestra de 366 estudiantes de distintas escuelas profesionales del I y VIII ciclo de estudios, de los cuales 232 estudiantes corresponden al primer ciclo y 134 estudiantes corresponden al octavo ciclo.

Los principales resultados obtenidos ponen de manifiesto que el Eje Transversal de Ética y Valores ha influido en el desarrollo de los valores de conformidad y reconocimiento en los estudiantes de las distintas escuelas profesionales, mostrando, los estudiantes del VIII ciclo, una actitud más analítica de las normas sociales convencionales. Los estudiantes del I ciclo evidencian una mayor necesidad de ser reconocidos y admirados por los demás. Los valores de soporte, independencia, benevolencia y liderazgo no presentan diferencias significativas entre los grupos de estudiantes del I ciclo y los del VIII ciclo, pero se observa un ligero incremento en los valores de independencia y liderazgo a favor de los estudiantes del VIII ciclo.

Los resultados por género, señalan que para los estudiantes varones, la escala que presenta diferencias significativas, es la escala de reconocimiento. Para las estudiantes mujeres las escalas que presentan diferencias son las de conformidad y reconocimiento.
\end{abstract}

Palabras clave: valores interpersonales, adolescencia, sistema de valores, valores

\begin{abstract}
This research enables to know the impact of the Transversal Axis of Ethics and Values, in the development of interpersonal values in the students of the first and eight cycle of a private university. The aforementioned axis is complementary to the academic education in aspects related to values like solidarity, leadership, support, among others; and it has as the objective to contribute to human education. For this research it was used the Survey of Interpersonal Values of Leonard Gordon, applying it to a sample of 366 students from different professional schools from the 1st and VIII cycle of studies; from which 232 students correspond to the first cycle and 134 students correspond to the eight cycle.

The main obtained results clearly show that the Transversal Axis of Ethics and Values has influenced in the development of compliance and recognition in the students of different professional schools. The VIII cycle students show a more analytic attitude toward conventional social rules. The 1st cycle students show a greater necessity to be recognized and admired by others. The values of support, independence, benevolence and leadership do not present significant differences between the groups of students from the 1st and the VIII cycle; but it is observed a slight increase in the values of independence and leadership in favor of the VIII cycle students.

The results by gender point out that for male students the scale that presents significant differences is the one from recognition. For the female students the scales with differences are the ones from compliance and recognition.
\end{abstract}

Key words: interpersonal values, adolescence, value system, values.

* Magíster en Psicología con mención en Prevención e Intervención en Niños y Adolescentes. Candidata al Doctorado en Psicología por la Universidad Femenina del Sagrado Corazón. Lima-Perú. Coach Ontológico, Pontificia Universidad Católica del Perú. grgrtp@yahoo.com 


\section{INTRODUCCIÓN}

Los valores tienen diferentes acepciones pero se entienden, por lo general, como las creencias que tienen las personas acerca de lo que consideran importante en la vida, tanto a nivel ético como moral, proporcionando la base para hacer juicios o elecciones acorde con el sistema personal de valores (Montuschi, 2008).

En el proceso de desarrollo adquirimos valores a través del proceso de socialización, cuando aprendemos pautas de comportamiento en la familia, en el colegio, en el barrio; por lo tanto, los valores se verán influenciados por la cultura, la educación, la familia, los medios de comunicación e información, entre otros. La consolidación o modificación de nuestro sistema permanecerá implícito en el comportamiento y servirá para tomar decisiones desde lo moral o desde la ética.

Sin embargo hablamos de valores positivos o valores negativos, infiriendo la polaridad de los valores. Pero además, no todos los valores tienen el mismo significado sino que están ubicados en valores superiores e inferiores, y vemos que existe una jerarquía de valores, independiente para cada persona que le sirve para orientar su comportamiento y le facilita tomar decisiones desde lo ético o moral, aspectos importantes que le permiten guiar su existencia y todo lo que considere importante para él como individuo o como integrante de un grupo social.

Por lo tanto, la importancia de los valores individuales o personales radica en el hecho de que se convierten en el vehículo motivador de nuestro comportamiento y el facilitador de la toma de decisiones.

Lawrence Kohlberg (Montuschi, 2005, p. 8) señala que en cada etapa de la vida de los jóvenes habrán de predominar ciertos valores:

- En el "nivel preconvencional" (5-8 años) el niño responde a las normas culturales y/o sociales interpretándolas de acuerdo a las consecuencias de su acción o respuesta (castigo o premio) o de acuerdo al poder físico de la autoridad que emite las normas. Es una forma de razonamiento moral egocéntrica. Los valores predominantes en este nivel son la obediencia a la autoridad y la reciprocidad.
- El comportamiento en el "nivel convencional" (8-14 años) es actuar de acuerdo con las expectativas del grupo que uno integra sin tomar en cuenta las consecuencias. Pero además es imperativo para el niño, mantener el orden en la sociedad y ello implica comprender las normas y leyes. La base de este nivel es relacionar los puntos de vista de todos. Posteriormente, lo fundamental es hacer lo correcto cumpliendo con el deber de cada uno como miembro de la sociedad, que conlleve a mantener el orden social y las leyes.

- En el siguiente nivel, "posconvencional y de principios" (16-18 ó hasta 25 años), se busca definir principios y valores morales que están por encima de las personas. Aquí lo correcto es sostener los valores, derechos básicos y contratos legales de la sociedad aunque ellos entren en conflicto con las reglas y leyes del grupo al cual se pertenece. La persona es consciente de la gran diversidad de valores y opiniones que tiene la gente, y el cual es relativo a su grupo de pertenencia. Aquí lo que se pretende es "el mayor bien para el mayor número", por ejemplo: la libertad, la vida, entre otros que deben mantenerse siempre. El comportamiento se ajusta y se guía por principios éticos universales basados en la justicia, el valor y la igualdad de todos y el respeto a la dignidad. El principio moral es que las personas no son medios sino fines en sí mismas.

Los valores se revelan con especial claridad cuando se está en la necesidad de efectuar una elección importante y difícil. Un sistema de valores claro y definido produce bienestar, puesto que hace sumamente fácil tomar decisiones y hacer elecciones.

Otro autor como Gordon (citado por Barsallo Saldaña, 2005), define al valor como todo aquello que la persona considera importante y que se constituye en una guía de su conducta, lo cual influirá en su nivel de ajuste personal, social, familiar y profesional. Por su parte, Gibson (citado por Angulo Zavaleta, 2001) afirma que los valores son normas y creencias que una persona adquiere a una temprana edad y que se constituyen en parte importante de sus pensamientos, empleándolos para enfrentar una situación en la que 
hay que tomar decisiones. También, Adela Cotrina (2001) considera que los valores son las cualidades o virtudes morales que caracterizan a los integrantes de una sociedad, distinguiéndose como personas íntegras para desempeñar cualquier oficio, cargo o profesión, con excelencia cualitativa, en lo moral, lo ético y en su propia capacidad personal.

La formación de los valores se da través de la interacción social, de la relación que entabla el individuo con su contexto. Es un proceso de aprendizaje dinámico, la facilidad con la que un individuo altera el aprendizaje dependerá de la importancia que para la persona tenga el valor afectado por la nueva información.

Por otro lado, Leonard Gordon (1977) explica que los valores relacionados con lo que las personas hacen y el modo cómo lo hacen están referidos a los valores interpersonales; aquéllas están influidas por el sistema de valores de cada individuo, lo cual determina la compatibilidad o incompatibilidad de los valores individuales con los valores del grupo y el modo como se relaciona con los demás. Según este autor estos valores serían: soporte (ser tratado con comprensión, amabilidad), conformidad (hacer lo que es socialmente correcto), reconocimiento (ser respetado y admirado), independencia (ser libre para tomar decisiones por sí mismo), benevolencia (hacer cosas por los demás) y liderazgo (tener autoridad y poder).

La familia es la principal formadora de los valores, es la que da las bases para su desarrollo posterior en el ámbito educativo. Siendo la universidad la encargada de consolidar esta formación iniciada en la niñez, es responsable de formar profesionales con un alto nivel académico y técnico, así como de desarrollar valores en sus egresados para ser insertados en la sociedad como ciudadanos responsables, competentes y comprometidos con el bien común. De este modo la universidad se convierte en el espacio de desarrollo del "ser" individual y colectivo a través de la práctica y ejercicio de los valores ligada a la integralidad de las personas.

La presente investigación se llevó a cabo en una universidad ubicada en la ciudad de Chimbote, Perú. Esta institución universitaria se visualiza como una comunidad universitaria, de docentes, estudiantes y trabajadores que centra su accionar en el servicio de calidad al estudiante, desarrollando sus fines de enseñanza, investigación y proyección social, en continuo proceso de innovación, crecimiento y mejora permanente, como una organización de aprendizaje con responsabilidad social universitaria.

En 1997 se incorporan cursos de Ética y Valores y de Pastoral Universitaria con el apoyo de la Diócesis de Chimbote. Algunos años después, con el apoyo de la Universidad de Piura, se organizó el currículo de Ética y Valores eje de competencias especializadas para la formación profesional- como un eje transversal curricular. El propósito fue crear una identidad corporativa que genere una cultura de valores en el actuar de la comunidad universitaria en base al humanismo cristiano y tomando para sí el paradigma de la autorrealización. El eje transversal de Ética y Valores es un eje de asignaturas especializadas, de desarrollo ético, psicológico, antropológico y social. Las asignaturas fueron de carácter obligatorio y con un valor de dos créditos por asignatura y se llevaron del I al VIII ciclo de estudios.

Chimbote es una ciudad situada al noreste del Perú. Las condiciones climáticas desérticas pertenecen a la clasificación de desiertos sub-tropicales. La temperatura oscila de $28^{\circ}$ a $13^{\circ}$ grados. El vocablo Chimbote proviene de dos voces: "CHIMBA", que según el vocabulario de Gonzales Holguín (citado por Bazán Blass, 2003) significa "la otra parte o banda del río o quebrada o acequia o casa larga atravesada"; y "BOTE", denominación de pequeña embarcación a remos. La palabra Chimbote aparece aproximadamente por el año 1760 oficializándose por el 1860. A los oriundos, inicialmente, se les denominó "Chimbadores"-también caleteros-y décadas más, chimboteros, hasta mediados de 1950, en que se generaliza la palabra chimbotano.

Desde la época de los conquistadores españoles, los lugareños atravesaban o ayudaban a cruzar a nado o a pie el río grande "Mayao" o "Santa" como se le conoce ahora.

Por la década del 40 al 50 se presentan aglomeraciones sorpresivas de gentes venidas de las serranías de Ancash, Cajamarca y La Libertad, así como del norte peruano, gente dedicada a la agricultura. La inmigración de "todas las sangres" formó una sociedad pluricultural. 
Los primeros pobladores de Chimbote, pescadores huanchaqueros, trajeron su misticismo representado en la devoción a su Santo Patrón San Pedro, y con ellos sus costumbres, los que fueron formando las características del poblador de la zona, la cual transmitió a las generaciones siguientes de pobladores ya oriundos del lugar.

\section{OBJETIVOS}

\section{Objetivo General}

Determinar la influencia del Eje Transversal de Ética y Valores en el desarrollo de los Valores Interpersonales en los estudiantes del I y VIII ciclo de las distintas escuelas profesionales y establecer las diferencias significativas.

\section{Objetivos Específicos}

1. Describir el nivel de desarrollo de las escalas de los Valores Interpersonales de soporte, conformidad, reconocimiento, independencia, benevolencia y liderazgo; en estudiantes del I y VIII ciclo de las distintas escuelas profesionales.

2. Conocer las diferencias significativas en el desarrollo de los Valores Interpersonales de los estudiantes del I y VIII ciclo de las distintas escuelas profesionales.

3. Conocer las diferencias significativas en el desarrollo de los Valores Interpersonales en los estudiantes de género femenino del I y VIII ciclo de las distintas escuelas profesionales.

4. Conocer las diferencias significativas en el desarrollo de los Valores Interpersonales en los estudiantes de género masculino del I y VIII ciclo de las distintas escuelas profesionales.

\section{HIPÓTESIS}

\section{Hipótesis Generales}

HG1: Existen diferencias significativas en el desarrollo de los Valores Interpersonales en los estudiantes del I y VIII ciclo de las distintas escuelas profesionales.

HG2: Existen diferencias significativas en el desarrollo de los Valores Interpersonales, de acuerdo al género, en los estudiantes del Iy VIII ciclo de las distintas escuelas profesionales.

\section{Hipótesis Específicas}

Ho: No existen diferencias significativas en el desarrollo de la escala Soporte de los Valores Interpersonales en los estudiantes del I y VIII ciclo de las distintas escuelas profesionales.

$\mathrm{H} 1$ : Existen diferencias significativas en el desarrollo de la escala Soportede los Valores Interpersonales en los estudiantes del I y VIII ciclo de las distintas escuelas profesionales.

Ho: No existen diferencias significativas en el desarrollo de la escala Conformidad de los Valores Interpersonales en los estudiantes del I y VIII ciclo de las distintas escuelas profesionales.

$\mathrm{H} 2$ : Existen diferencias significativas en el desarrollo de la escala Conformidad de los Valores Interpersonales en los estudiantes del I y VIII ciclo de las distintas escuelas profesionales.

Ho: No existen diferencias significativas en el desarrollo de la escala Reconocimiento de los Valores Interpersonales en los estudiantes del Iy VIII ciclo de las distintas escuelas profesionales.

H3: Existen diferencias significativas en el desarrollo de la escala Reconocimiento de los Valores Interpersonales en los estudiantes del I y VIII ciclo de las distintas escuelas profesionales.

Ho: No existen diferencias significativas en el desarrollo de la escala Independencia de los Valores Interpersonales en los estudiantes del 1 ro y VIII ciclo de las distintas escuelas profesionales.

$\mathrm{H} 4$ : Existe diferencias significativas en el desarrollo de la escala Independencia de los Valores Interpersonales en los estudiantes del I y VIII ciclo de las distintas escuelas profesionales.

Ho: No existen diferencias significativas en el desarrollo de la escala Benevolenciade los Valores Interpersonales en los estudiantes del I y del VIII ciclo de las distintas escuelas profesionales.

H5: Existen diferencias significativas en el desarrollo de la escala Benevolencia de los Valores Interpersonales en los estudiantes del I y del VIII ciclo de las distintas escuelas profesionales.

Ho: No existen diferencias significativas en el desarrollo de la escala Liderazgo de los Valores Interpersonales en los estudiantes del I y VIII ciclo de las distintas escuelas profesionales.

H6: Existen diferencias significativas en el desarrollo de la escala Liderazgo de los Valores Interpersonales en los estudiantes del I y VIII ciclo de las distintas escuelas profesionales. 
Ho: No existen diferencias significativas en el desarrollo de la escala Soporte de los Valores Interpersonales de acuerdo al género de los estudiantes del I y VIII ciclo de las distintas escuelas profesionales.

$\mathrm{H7}$ : Existen diferencias significativas en el desarrollo de la escala Soportede los Valores Interpersonales, de acuerdo al género, en los estudiantes del l y VIII ciclo de las distintas escuelas profesionales.

Ho: No existen diferencias significativas en el desarrollo de la escala Conformidad de los Valores Interpersonales, de acuerdo al género, en los estudiantes del I y del VIII ciclo de las distintas escuelas profesionales.

H8: Existen diferencias significativas en el desarrollo de la escala Conformidad de los Valores Interpersonales, de acuerdo al género, en los estudiantes del I y VIII ciclo de las distintas escuelas profesionales.

Ho: No existen diferencias significativas en el desarrollo de la escala Reconocimiento de los Valores Interpersonales, de acuerdo al género, en los estudiantes del I y el VIII ciclo de las distintas escuelas profesionales.

H9: Existen diferencias significativas en el desarrollo de la escala Reconocimiento de los Valores Interpersonales, de acuerdo al género, en los estudiantes del I y VIII ciclo de las distintas escuelas profesionales.

Ho: No existen diferencias significativas en el desarrollo de la escala Independencia de los Valores Interpersonales, de acuerdo al genero, en los estudiantes del I y VIII ciclo de las distintas escuelas profesionales.

$\mathrm{H} 10$ : Existen diferencias significativas en el desarrollo de la escala Independencia de los Valores Interpersonales, de acuerdo al género, en los estudiantes del I y VIII ciclo de las distintas escuelas profesionales.

Ho: No existen diferencias significativas en el desarrollo de la escala Benevolenciade los Valores Interpersonales, de acuerdo al género, en los estudiantes del I y VIII ciclo de las distintas escuelas profesionales.

$\mathrm{H} 11$ : Existen diferencias significativas en el desarrollo de la escala Benevolencia de los Valores Interpersonales, de acuerdo al género, en los estudiantes del I y VIII ciclo de las distintas escuelas profesionales.
Ho: No existen diferencias significativas en el desarrollo de la escala Liderazgo de los Valores Interpersonales, de acuerdo al género, en los estudiantes del I y VIII ciclo de las distintas escuelas profesionales.

$\mathrm{H} 12$ : Existen diferencias significativas en el desarrollo de la escala Liderazgo de los Valores Interpersonales, de acuerdo al género, en los estudiantes del I y del VIII ciclo de las distintas escuelas profesionales.

\section{MÉTODO}

Es un trabajo de tipo descriptivo-comparativo. En él se analizan los valores interpersonales de los estudiantes del primer y octavo ciclo de las distintas carreras profesionales participantes en el presente estudio.

\section{Participantes}

La muestra estuvo conformada por un total de 366 estudiantes; de los cuales 232 corresponden a estudiantes del primer ciclo y 134 a estudiantes del octavo ciclo de estudios de las distintas escuelas profesionales de la universidad (ver Tabla 1), y dentro de los rangos de edad comprendidos entre los 17 a 21 años de edad, para el primer ciclo, y de 21 a 25 años de edad para los estudiantes del octavo ciclo (ver Tabla 2).

La muestra fue seleccionada por criterios de inclusión, considerando los siguientes: ser estudiante matriculado en el primer y octavo ciclo de las distintas carreras profesionales de la universidad y asistir regularmente a clases; estar entre los rangos de edad de 17 a 25 años; y estudiantes de ambos sexos.

La universidad se encuentra ubicada en la zona norte del país, en la localidad de Chimbote; los estudiantes proceden de familias afincadas en la localidad, las cuales se desempeñan en actividades relacionadas a la pesca en sus distintas variantes.

\section{Materiales y procedimiento}

El instrumento utilizado fue el Cuestionario de Valores Interpersonales de Leonard Gordon (1977) Survey of Interpersonal Values, S.I.V.-, el cual ha sido elaborado con las técnicas del análisis factorial y como un cuestionario de elección forzada en triadas (30 triadas que en conjunto suman 90 ítems). EI 
cuestionario permite apreciar y comparar la importancia que una persona da a los valores, actitudes o aspectos de sus relaciones con los demás. Evalúa los siguientes valores interpersonales: soporte, conformidad, reconocimiento, independencia, benevolencia y liderazgo.

El procesamiento estadístico se realizó mediante el siguiente procedimiento: 1) aplicación del instrumento a los estudiantes de ambos sexos de las escuelas profesionales seleccionadas; 2) la información recolectada se organizó en el programa estadístico Excel, realizando cuadros y gráficos; 3) el procesamiento de la información se hizo utilizando el SPSS15; 4) Para la contrastación de hipótesis para determinar las diferencias de medias se utilizó la Normal estándar (Z) con un nivel de confianza del $0,95 \%$.

Tabla 1

Frecuencia de la muestra de estudiantes del I y VIII ciclo de las distintas escuelas profesionales de una universidad privada

\begin{tabular}{|c|c|c|c|}
\hline \multirow[t]{2}{*}{ Escuelas } & \multicolumn{2}{|c|}{ Ciclo } & \multirow[t]{2}{*}{ Total } \\
\hline & $1^{\circ}$ Ciclo & $8^{\circ}$ Ciclo & \\
\hline Enfermería & 35 & 13 & 48 \\
\hline Obstetricia & 4 & 17 & 21 \\
\hline Farmacia & 8 & 6 & 14 \\
\hline Odontología & 26 & 12 & 38 \\
\hline Contabilidad & 37 & 15 & 52 \\
\hline Administración & 38 & 11 & 49 \\
\hline Educación & 34 & 10 & 44 \\
\hline Derecho & 33 & 31 & 64 \\
\hline Ing. Sistemas & 17 & 19 & 36 \\
\hline Total & 232 & 134 & 366 \\
\hline
\end{tabular}

Tabla 2

Frecuencia de las edades de los estudiantes del l y VIII ciclo de las distintas escuelas profesionales de una universidad privada

\begin{tabular}{lccccc}
\hline $\begin{array}{l}\text { I ciclo } \\
\text { edad }\end{array}$ & Frecuencia & Porcentaje & VIII cicloedad & Frecuencia & Porcentaje \\
\hline 17 & 43 & 18.53 & 21 & 1 & 0.75 \\
18 & 90 & 38.79 & 22 & 56 & 41.79 \\
19 & 35 & 15.09 & 23 & 54 & 40.30 \\
20 & 9 & 3.88 & 24 & 16 & 11.94 \\
21 & 55 & 23.71 & 25 & 7 & 5.22 \\
Total & 232 & 100.00 & TOTAL & 134 & 100.00 \\
\hline
\end{tabular}




\section{RESULTADOS}

La Tabla 3 detalla las medidas estadísticas para la inferencia de promedios obtenidos por los estudiantes del I y VIII ciclo en las escalas de soporte (S), conformidad $(C)$, reconocimiento $(R)$, independencia (I), benevolencia (B) y liderazgo (L), de los valores interpersonales de las diferentes escuelas profesionales. Esta tabla nos revela que el promedio aritmético en las distintas escalas se ubica en un nivel medio; observándose un ligero incremento en el promedio para las escalas de conformidad, benevolencia y liderazgo a favor de los estudiantes del I ciclo de estudios.

Tabla 3

Medidas estadísticas para la inferencia de promedios por niveles de los estudiantes del ly VIII ciclo

\begin{tabular}{|c|c|c|c|c|c|c|c|c|c|c|c|c|}
\hline \multirow[t]{2}{*}{ Estadígrafos } & \multicolumn{6}{|c|}{ Primer Ciclo } & \multicolumn{6}{|c|}{ Octavo Ciclo } \\
\hline & $\mathbf{S}$ & C & $\mathbf{R}$ & I & B & $\mathbf{L}$ & S & C & $\mathbf{R}$ & $\mathbf{I}$ & B & $\mathbf{L}$ \\
\hline $\mathbf{N}$ & 232 & 232 & 232 & 232 & 232 & 232 & 134 & 134 & 134 & 134 & 134 & 134 \\
\hline $\begin{array}{l}\text { Promedio } \\
\text { Aritmético }\end{array}$ & 15.56 & 16.76 & 10.25 & 14.34 & 19.06 & 14.03 & 15.58 & 15.34 & 11.78 & 14.55 & 18.65 & 13.96 \\
\hline $\begin{array}{l}\text { Desviación } \\
\text { Estándar }\end{array}$ & 4.09 & 4.705 & 3.733 & 4.755 & 5.016 & 5.285 & 4.135 & 4.613 & 4.311 & 5.084 & 4.827 & 4.929 \\
\hline Varianza & 16.72 & 22.14 & 13.94 & 22.61 & 25.16 & 27.93 & 17.1 & 21.28 & 18.58 & 25.84 & 23.3 & 24.3 \\
\hline
\end{tabular}

La Tabla 4 muestra las medidas estadísticas para la inferencia de promedios obtenidos por los estudiantes varones del I y VIII ciclo en las escalas de soporte, conformidad, reconocimiento, independencia, benevolencia y liderazgo, de los valores interpersonales de las diferentes escuelas profesionales, observándose que el promedio aritmético obtenido, por ambos grupos de estudiantes varones, los ubica en un nivel medio. Sin embargo las escalas de soporte, conformidad, independencia y benevolencia presentan un ligero incremento a favor de los estudiantes varones del I ciclo; siendo las escalas de reconocimiento y liderazgo a favor de los estudiantes varones del VIII ciclo.

Tabla 4

Medidas estadísticas para la inferencia de promedios por niveles de los estudiantes varones del ly VIII ciclo

\begin{tabular}{|c|c|c|c|c|c|c|c|c|c|c|c|c|}
\hline \multirow[t]{2}{*}{ Estadígrafos } & \multicolumn{6}{|c|}{ Primer ciclo } & \multicolumn{6}{|c|}{ Octavo ciclo } \\
\hline & $S$ & C & $\mathbf{R}$ & $\mathbf{I}$ & B & $\mathbf{L}$ & $\mathbf{S}$ & C & $\mathbf{R}$ & $\mathbf{I}$ & B & $\mathbf{L}$ \\
\hline $\mathbf{N}$ & 99 & 99 & 99 & 99 & 99 & 99 & 58 & 58 & 58 & 58 & 58 & 58 \\
\hline $\begin{array}{l}\text { Promedio } \\
\text { Aritmético }\end{array}$ & 15.63 & 16.13 & 10.9 & 14.21 & 18.34 & 14.79 & 14.88 & 15.24 & 12.57 & 13.41 & 18.02 & 15.53 \\
\hline $\begin{array}{l}\text { Desviación } \\
\text { Estándar }\end{array}$ & 4.256 & 4.823 & 3.908 & 4.832 & 4.943 & 4.773 & 4.143 & 4.688 & 4.453 & 5.195 & 4.643 & 5.378 \\
\hline Varianza & 18.11 & 23.26 & 15.28 & 23.35 & 24.43 & 22.78 & 17.16 & 21.98 & 19.83 & 26.98 & 21.56 & 28.92 \\
\hline
\end{tabular}


La Tabla 5 detalla las medidas estadísticas para la inferencia de promedios obtenidos por las estudiantes mujeres del I y VIII ciclo en las escalas de soporte, conformidad, reconocimiento, independencia, benevolencia y liderazgo, de los valores interpersonales de las diferentes escuelas profesionales. Esta tabla nos indica que el promedio aritmético obtenido por ambos grupos de estudiantes mujeres las ubica en un nivel medio. Observándose un ligero incremento en las escalas de conformidad, benevolencia y liderazgo a favor del grupo de estudiantes mujeres del I ciclo; y en las escalas de soporte, reconocimiento e independencia a favor del grupo de estudiantes mujeres del VIII ciclo.

Tabla 5

Medidas estadísticas para la inferencia de promedios por niveles de las estudiantes mujeres del ly VIII ciclo

\begin{tabular}{|c|c|c|c|c|c|c|c|c|c|c|c|c|}
\hline \multirow[t]{2}{*}{ Estadígrafos } & \multicolumn{6}{|c|}{ Primer Ciclo } & \multicolumn{6}{|c|}{ Octavo Ciclo } \\
\hline & $\mathbf{S}$ & C & $\mathbf{R}$ & I & B & $\mathbf{L}$ & $\mathbf{S}$ & C & $\mathbf{R}$ & I & B & $\mathbf{L}$ \\
\hline $\mathbf{N}$ & 133 & 133 & 133 & 133 & 133 & 133 & 76 & 76 & 76 & 76 & 76 & 76 \\
\hline $\begin{array}{l}\text { Promedio } \\
\text { Aritmético }\end{array}$ & 15.5 & 17.23 & 9.767 & 14.44 & 19.6 & 13.46 & 16.17 & 15.33 & 10.97 & 15.47 & 19.29 & 12.76 \\
\hline $\begin{array}{l}\text { Desviación } \\
\text { Estándar }\end{array}$ & 3.976 & 4.579 & 3.535 & 4.712 & 5.021 & 5.586 & 4.062 & 4.569 & 4.156 & 4.936 & 4.865 & 4.255 \\
\hline Varianza & 15.81 & 20.96 & 12.5 & 22.2 & 25.21 & 31.2 & 16.5 & 20.87 & 17.27 & 24.36 & 23.67 & 18.1 \\
\hline
\end{tabular}

En la Tabla 6 se observa que el valor $Z$ es igual a 2,82 cuyo valor es mayor que la $Z$ Tabular $(1.96$ al nivel de confianza de 0.05 ) por lo que se deduce que existen diferencias significativas en el desarrollo de la escala de conformidad de los valores interpersonales en los estudiantes del I y VIII ciclo de las distintas escuelas profesionales de la universidad.

Tabla 6

Diferencia de medias del valor interpersonal de conformidad en estudiantes varones y mujeres del I y VIII ciclo de una Universidad privada

\begin{tabular}{rrrrrrr}
\hline Ciclo & $\mathrm{N}$ & $\begin{array}{r}\text { Promedio } \\
\text { Aritmético }\end{array}$ & $\begin{array}{r}\text { Desviación } \\
\text { Estándar }\end{array}$ & $\begin{array}{r}\text { Varianza } \\
(+/-) 1,96\end{array}$ & Valor Z & Decisión Z \\
\hline I & 232 & 16,76 & 4,705 & 22,14 & 2,82 & Significativo \\
VIII & 134 & 15,34 & 4,613 & 21,28 & & \\
\hline
\end{tabular}

En la Tabla 7 se observa que el valor $Z$ es igual a 3,420 cuyo valor es mayor que la Z Tabular ( 1.96 al nivel de confianza de 0.05) por lo que se deduce que existen diferencias significativas en el desarrollo de la escala de reconocimiento de los valores interpersonales en los estudiantes del I y VIII ciclo de las distintas escuelas profesionales de la universidad. 
Tabla 7

Diferencia de medias del valor interpersonal de reconocimiento en estudiantes varones y mujeres del I y VIII ciclo

\begin{tabular}{lrrrrrr}
\hline Ciclo & $\mathrm{N}$ & $\begin{array}{r}\text { Promedio } \\
\text { Aritmético }\end{array}$ & $\begin{array}{r}\text { Desviación } \\
\text { Estándar }\end{array}$ & $\begin{array}{r}\text { Varianza } \\
(+/-) 1,96\end{array}$ & Valor Z & Decisión Z \\
\hline I & 232 & 10,25 & 3,733 & 13,94 & 3,420 & Significativo \\
VIII & 134 & 11,78 & 4,311 & 18,58 & & \\
\hline
\end{tabular}

En la Tabla 8 los resultados obtenidos señalanque el valor $Z$ es igual a 2,88 el cual es mayor que la $Z$ Tabular (1.96 al nivel de confianza de 0.05 ) por lo que se infiere que existen diferencias significativas en el desarrollo de la escala de conformidad de los valores interpersonales en las estudiantes mujeres del I y VIII ciclo de las distintas escuelas profesionales.

Tabla 8

Diferencia de medias del valor interpersonal de conformidad en estudiantes mujeres dell y VIII ciclo

\begin{tabular}{rrrrrrr}
\hline Ciclo & N & $\begin{array}{r}\text { Promedio } \\
\text { Aritmético }\end{array}$ & $\begin{array}{r}\text { Desviación } \\
\text { Estándar }\end{array}$ & $\begin{array}{r}\text { Varianza } \\
(+/-) 1,96\end{array}$ & Valor Z & Decisión Z \\
\hline I & 133 & 17,23 & 4,579 & 20,96 & 2,88 & Significativo \\
VIII & 76 & 15,33 & 4,569 & 20,87 & & \\
\hline
\end{tabular}

En la Tabla 9 los resultados obtenidos señalanque el valor Z es igual a 2,19 el cual es mayor que la Z Tabular (1.96 al nivel de confianza de 0.05 ) por lo que se deduce que existen diferencias significativas en el desarrollo de la escala de reconocimiento de los valores interpersonales en las estudiantes mujeres del I y VIII ciclo de las distintas escuelas profesionales.

Tabla 9

Diferencia de medias del valor interpersonal de reconocimiento en estudiantes mujeres del ly VIII ciclo

\begin{tabular}{lrrrrrr}
\hline Ciclo & N & $\begin{array}{r}\text { Promedio } \\
\text { Aritmético }\end{array}$ & $\begin{array}{r}\text { Desviación } \\
\text { Estándar }\end{array}$ & $\begin{array}{r}\text { Varianza } \\
(+/-) 1,96\end{array}$ & Valor Z & Decisión Z \\
\hline I & 133 & 9,767 & 3,53 & 12,5 & 2,19 & Significativo \\
VIII & 76 & 10,97 & 4,156 & 17,27 & & \\
\hline
\end{tabular}


En la Tabla 10 los resultados obtenidos señalan que el valor de Z es igual a 2,37 el cual es mayor que Z Tabular (1.96 al nivel de confianza de 0.05 ) por lo que se infiere que existen diferencias significativas en el desarrollo de la escala de reconocimiento de los valores interpersonales en los estudiantes varones del I y VIII ciclo de las distintas escuelas profesionales.

Tabla 10

Diferencias de medias del valor interpersonal de reconocimiento en estudiantes varones del ly VIII ciclo

\begin{tabular}{lrrrrrr}
\hline Ciclo & N & $\begin{array}{r}\text { Promedio } \\
\text { Aritmético }\end{array}$ & $\begin{array}{r}\text { Desviación } \\
\text { Estándar }\end{array}$ & $\begin{array}{r}\text { Varianza } \\
(+/-) 1,96\end{array}$ & Valor Z & Decisión Z \\
\hline I & 99 & 10,9 & 3,908 & 15,28 & 2,37 & Significativo \\
VIII & 58 & 12,57 & 4,453 & 19,83 & & \\
\hline
\end{tabular}

\section{DISCUSIÓN}

Al analizar los resultados alcanzados por ambos grupos -varones y mujeres estudiantes del I y VIII ciclo de una universidad privada, en la escala de soporte se aprecia que, en general, persiste un nivel medio, lo cual significa que existe la necesidad de comprensión afectiva y protectora en un nivel intermedio.

La diferencia de promedios entre ambos grupos de estudiantes, en las escalas de soporte, conformidad, reconocimiento, independencia, benevolencia y liderazgo, nos señala que las diferencias estadísticas no son significativas. Sin embargo, en el grupo de estudiantes varones del I y VIII ciclo, la diferencia de promedios, aunque no es significativa, nos indica una tendencia, para el grupo de estudiantes varones del VIII ciclo, a una menor necesidad de apoyo y comprensión de los demás. Estos hallazgos pueden estar muy relacionados al hecho de que a medida que se avanza en la carrera profesional, se evidencia mayor seguridad y madurez afectiva. Estos resultados se relacionan con los contenidos desarrollados por el Eje Transversal de Ética y Valores, los cuales a través del conocimiento de distintos aspectos como: descubrimiento del yo, conocerse y aceptarse como persona, autoconcepto, entre otros, busca desarrollar seguridad personal en los estudiantes. En el grupo de estudiantes mujeres del ly VIII ciclo, la diferencia estadística no es significativa; pero indica la tendencia, para el grupo de mujeres del I ciclo, a la necesidad de comprensión afectiva y protectora. En la investigación de Romero Lino y Villegas Chuman (2006) se halló un resultado similar, exponiendo que existe un predominio de la escala de soporte en el grupo de estudiantes del I ciclo quienes tienen mayor necesidad de contar con la consideración y aprobación de apoyo y comprensión de los demás.

La escala de conformidad, según Gordon (1977) está vinculada al grado de aceptación o no de las normas comunes de convivencia social, más desarrollada la actitud hacia lo que es socialmente correcto. En el grupo de estudiantes del I ciclo, así como en los del VIII ciclo hay predominio de un nivel Medio, es decir hay aceptación de las normas y reglas sociales consideradas adecuadas para la convivencia social.

Al analizar la diferencia de promedios de los estudiantes del I y VIII ciclo, en relación al valor interpersonal de conformidad, se observa que existen diferencias estadísticamente significativas; esto indicaría que los estudiantes del VIII ciclo, en general, luego de concluir con las actividades del Eje Transversal de Ética y Valores, evidencian una actitud analítica frente a las normas sociales y a lo que se considera socialmente correcto. Es decir, que asumen aquello que consideran socialmente correcto y rechazan aquello que no les parece. En el grupo de estudiantes varones del I y VIII ciclo, la diferencia de promedios señala que no hay diferencias estadísticas significativas, en la escala de conformidad.

Sin embargo, en el grupo de estudiantes mujeres del I y VIII ciclo, la diferencia de promedios señala que existen diferencias estadísticamente significativas, lo que podría indicar que las estudiantes mujeres del VIII ciclo son más cuestionadoras y analíticas de las 
normas sociales y de lo que se considera socialmente correcto.

Del mismo modo, estos hallazgos podemos compararlos con uno de los resultados del estudio de Sevillano López (2005) quien concluye que el valor de conformidad presenta diferencias significativas entre los estudiantes de enfermería y fisioterapia del I y VI ciclo.

En la escala de reconocimiento, la que hace referencia a la necesidad de ser admirado, de ser importante y que los demás reconozcan que es así, ambos grupos se ubican en el nivel medio, siendo los estudiantes del I ciclo quienes se movilizan en procura de ser admirados, estimados, de sentirse importantes y que los demás reconozcan que es así; a diferencia de los estudiantes del VIII ciclo quienes se revelan como más cautelosos y con menos necesidad de admiración y reconocimiento.

Al analizar la diferencia de promedios de los estudiantes del I y VIII ciclo, en general, encontramos que existen diferencias estadísticamente significativas; estos resultados señalan que existe unafuerte necesidad de ser admirado, de ser importante y que los demás reconozcan que es así.

En el grupo de estudiantes varones y mujeres, por separado, del I y VIII ciclo, la diferencia de promedios de la escala nos indica que existen diferencias estadísticamente significativas; es decir, para el grupo de estudiantes del I ciclo existe una fuerte necesidad, de ser admirados, de ser importante y ser reconocido por los demás. Se ven motivados socialmente a demostrar eficiencia y aptitud. Estos resultados son coincidentes con los encontrados por Sevillano López (2005) quien concluye que los estudiantes del I ciclo de Enfermería y Fisioterapia se encuentra en un nivel alto el valor de reconocimiento.

En los alumnos del VIII ciclo esta escala disminuye en su puntaje, esto puede estar relacionado con los contenidos del Eje Transversal de Ética y Valores busca desarrollar humildad (sencillez) en los beneficiarios.

Los resultados de la escala de independencia, la cual está en relación al ser individual, a la estimación de la libertad personal, a hacer las cosas a favor del propio criterio, indican que ambos grupos se ubican en un nivel Medio, esto quiere decir que existe una adecuada manifestación de la conducta colectiva e individualista.

Al analizar la diferencia de promedios de los estudiantes del I y VIII ciclo, se observa que no hay diferencias estadísticamente significativas.

De igual modo, en el grupo de estudiantes varones y mujeres, por separado, del I y VIII ciclo, la diferencia de promedios de la misma escala, indica que no existen diferencias estadísticas significativas, pero sí una tendencia en el grupo de estudiantes del VIII ciclo a la estimación de la libertad personal en desmedro de la tendencia a actuar pensando sólo en sus necesidades intereses, esta diferencia contribuye a una adecuada socialización para los estudiantes del VIII ciclo. Este resultado va en relación directa con el perfil del estudiante que busca desarrollar la universidad, un profesional tolerante frente a las diferencias de ideas, opiniones o puntos de vista. Estos hallazgos son coincidentes con los encontrados por Romero Lino y Villegas Chuman (2006) quienes concluyen que esta escala progresa favorablemente en la medida que se desarrollan otras habilidades como las académicas, las de formación profesional, entre otras, y que permite actuar con mayor autonomía a medida que éstas evolucionan.

En la escala de benevolencia observamos que en ambos grupos predomina el nivel alto, este resultado nos indica, según Gordon (1977), elevado nivel de actitud de amor hacia el prójimo. La diferencia de promedios de los estudiantes del I y VIII ciclo, nos muestra que no existen diferencias estadísticamente significativas.

Enel grupo de estudiantes varones y mujeres, por separado del I y VIII ciclo, se evidencia que no existen diferencias estadísticamente significativas.

A diferencia de las anteriores escalas, la escala de benevolencia es la que presenta resultados altos en ambos grupos, siendo mayor para el I ciclo, pero manteniéndose alto para los del VIII ciclo. Gordon (1977) señala que la benevolencia decae a medida que aumenta la preparación académica; podemos observar en el presente estudio, que la influencia del Eje Transversal de Ética y Valores ha contribuido a que los estudiantes del VIII ciclo aún muestren un alto 
grado de sensibilidad hacia las necesidades y problemas humanos. Estos resultados son coincidentes con los de Sevillano López (2005) quien concluye en su estudio que para los estudiantes del I de Enfermería y Fisioterapia el valor de soporte, reconocimiento, independencia, benevolencia y liderazgo se presenta en un nivel alto. Del mismo modo, para los estudiantes del VI ciclo de Enfermería y Fisioterapia el valor de conformidad, benevolencia y liderazgo se presenta en un nivel alto. Resultados similares concluye en su estudio Aguilar Castillo (2001), al señalar que los estudiantes de psicología de los tres primeros ciclos con alta autoestima poseen puntajes altos en los valores de liderazgo y benevolencia.

Finalmente, en la escala de liderazgo, la cual está relacionada, según Gordon, por la preferencia por las actitudes de mando y por toda situación que signifique tener autoridad sobre otros, podemos observar que ambos grupos se encuentran en el nivel medio, esto quiere decir que está presente la actitud de mando y de autoridad en un nivel medio. Si tomamos en cuenta que el valor de liderazgo es un comportamiento que implica tener la predisposición y el poder para tomar decisiones sobre los demás; así como ser capaz de persuadir, hallamos en ambos grupos un nivel medio con una mayor tendencia en el grupo de estudiantes del VIII ciclo. Estas apreciaciones se confirman con los estudios de Smith (citado por Aguilar Castillo, 2001), quien también halló un aumento de este valor en los estudiantes que culminan sus estudios profesionales.

Al analizar la diferencia de promedios de los estudiantes del I y VIII ciclo se observa que no existen diferencias estadísticamente significativas, pero sí indica una sutil tendencia para los estudiantes del VIII ciclo, por actitudes de mando y toma de decisiones con autoridad sobre otros.

En síntesis, en relación a los objetivos e hipótesis planteados se puede concluir -entre los aspectos más significativos-, que el Eje Transversal de Ética y Valores ha influido en el desarrollo de las escalas de Conformidady Reconocimiento, existiendo diferencias significativas en el desarrollo de la escala Conformidad (H2) de los Valores Interpersonales, entre los estudiantes del I y VIII Ciclo de las distintas escuelas profesionales, siendo los estudiantes del VIII los que asumen aquello que consideran socialmente correcto y rechazan aquello que no les parece, además se muestran más analíticos de las normas consideradas socialmente correctas, en comparación con los estudiantes del I ciclo. Igualmente existen diferencias significativas en el desarrollo de la escala Reconocimiento (H3) entre los estudiantes mencionados, siendo los estudiantes del I ciclo quienes revelan una fuerte necesidad de ser admirados, de ser importante y que los demás los reconozcan.

Según el género, para las mujeres, existen diferencias significativas en el desarrollo de la escala Conformidad (H8) de los Valores Interpersonales, entre las estudiantes del I y VIII Ciclo de las distintas escuelas profesionales, siendo el grupo del VIII ciclo quienes se sujetan más a las normas sociales que consideran correctas y rechazan las que no lo consideran así, y se muestran más analíticas en relación a estas normas, en comparación con las alumnas del I ciclo. Del mismo modo, en relación al grupo de varones la escala de Reconocimiento $(\mathrm{H} 9)$ presenta diferencias significativas con respecto al grupo de estudiantes del I y VIII ciclo, siendo los del I ciclo quienes revelan una fuerte necesidad de ser reconocidos por lo que hacen, en comparación con los estudiantes del VIII ciclo de las distintas escuelas profesionales.

Los valores de soporte, independencia, benevolencia y liderazgo no presentan diferencias significativas entre los grupos de estudiantes del I y VIII ciclo; sin embargo se puede apreciar un ligero incremento en los estudiantes que culminan sus estudios profesionales.

Consideramos que es importante seguir realizando investigaciones en el tema de los valores, relacionadas con otras variables como psicológicas y sociales. Los valores constituyen un proceso personal que se desarrolla a través de la vida; en cada momento tomamos decisiones analizando cada situación y valorando nuestros actos en relación a una escala de valores que nos permiten enfrentarnos en el futuro. Los valores son la parte que le da estructura central al ser humano, especialmente aquellos valores de orden superior.

Los resultados hallados permiten sugerir la promoción de una revisión de la estructura curricular del Eje Transversal de Ética y Valores, con la finalidad de realizar los cambios necesarios para potencializar 
los resultados concordantes con el perfil del egresado que espera obtener la Universidad Los Ángeles de Chimbote.

Asimismo sería importante realizar programas de cultura organizacional que incluyan el fortalecimiento de los valores interpersonales en la comunidad universitaria: funcionarios, docentes, personal administrativo, jefes de departamento, etc., requisito necesario para la formación humana y ética en todos los niveles, que permita contribuir al cumplimiento del paradigma de la autorrealización, aspecto principal en su política de trabajo, basada en la Doctrina Social de la Iglesia.

\section{REFERENCIAS}

Aguilar Castillo, B. (2001). Estudio comparativo de los valores interpersonales con alumnos con alta y baja autoestima de los tres primeros ciclos de la Escuela de Psicología de la Universidad Señor de Sipán-Trujillo (Tesis de licenciatura en Psicología). Universidad Señor de Sipán, TrujilloPerú.

Angulo Zavaleta, I. (2001). Estudio comparativo de los valores interpersonales en trabajadores remunerados y voluntarios de los centros asistenciales de EsSalud de la Ciudad de Trujillo (Tesis de licenciatura en Psicología). Universidad César Vallejo, Trujillo-Perú.

Barsallo Saldaña, L. (2005). Valores interpersonales en estudiantes del VII ciclo de la Facultad de Medicina de la Universidad Privada San Pedro 2005-I. (Tesis de licenciatura en Psicología). Universidad Privada San Pedro, Chimbote, Perú.
Bazán Blass, F. (2003). Historia de Chimbote. Chimbote, Perú: Editorial San MarcosULADECH.

Cotrina, A. (2001). El mundo de los valores. LimaPerú: Editorial Andarín.

Gordon, Leonard (1977). Cuestionario de valores interpersonales. Madrid: Editorial TEA.

Montuschi, L. (2005). El aprendizaje moral de los individuos y las corporaciones: Las etapas de Kolberg, la cultura organizacional y los códigos de ética. Documento de trabajo. Universidad del CEMA. Recuperado de Base de Datos Ebscohost, FuenteAcadémica.

Montuschi, L. (2008). Los valores personales y los valores corporativos en el comportamiento ético en la empresa. Documento de trabajo. Buenos Aires: Universidad del CEMA. Recuperado de h t t p :// www.ucema.edu.ar/ / m/ ETICA_Y_NEGOCIOS_-_ARTICULOS/ Los_valores_personales y los_valores_corporativos.pdf

Romero Lino, J. y Villegas Chuman, C. (2006). Análisis comparativo de los valores interpersonales entre los estudiantes del primer ciclo respecto al onceavo de la Carrera Profesional de Psicología de la Universidad César Vallejo de la Ciudad de Trujillo (Tesis de licenciatura en Psicología). Universidad César Vallejo, Trujillo, Perú.

Sevillano López, M. (2005). Estudio comparativo de los niveles de los valores interpersonals en los estudiantes de las escuelas profesionales de Enfermería y Fisioterapia del I y VI ciclo del I.S.T. San Luis-Trujillo (Tesis de licenciatura en Psicología). Universidad César Vallejo, Trujillo, Perú. 